\title{
Botanicals and its extracts: A new drug discovery and development paradigm for chronic and age-related illnesses
}

\author{
Leodevico L Ilag* \\ Xerion Limited, Australia
}

\begin{abstract}
Advances in medical science have led to the cure and prevention of major diseases leading to longer lifespans. However, increasing lifespan has presented new medical challenges such as the rise of chronic and age-related illnesses. Chronic diseases are often associated with perturbations of multiple disease pathways and may require simultaneous modulation of multiple targets. Thus, the current paradigm of drug discovery and development based on a specific, single disease target may need to be complemented with a holistic systems-based approach. Although botanicals have historically been a source of new drugs, botanical extracts are not widely accepted in Western medicine. A relatively new regulatory approach with botanical extracts as a new class of drugs may pave the way for a new paradigm in discovering and developing therapies for chronic diseases.
\end{abstract}

\section{Introduction}

Drugs sourced from natural products such as microorganisms and plants have been the foundation of the pharmaceutical industry. Aspirin had its origins in the time of Hippocrates (460-370 BCE) where willow bark and salicylate-rich plants were used to reduce pain, fever and inflammation. The turning point came centuries later when Bayer scientists produced synthetic acetylsalicylic acid (aspirin) with the same effects as salicylate extracts. Another example is the fungus that Alexander Fleming identified in 1928, which had anti-bacterial activity. Howard Florey extracted the penicillin molecule paving the way for its large-scale commercial production and its revolutionary use to treat infections. Together Ernst Boris Chain, Fleming and Florey won the Nobel Prize in Physiology or Medicine in 1945.

In the last 40 years a drug discovery and development paradigm based on a single, drug target and its mechanism of action evolved. Advances in high-throughput screening, genomics and structurebased drug design validated and accelerated its progress. Examples include the discovery and development of more selective COX-2 (cyclooxygenase-2) inhibitors, with fewer side effects than aspirin; statins or inhibitors of HMG CoA reductase, the key enzyme in cholesterol biosynthesis for the treatment of hypercholesterolaemia; and anti-retroviral agents targeting the HIV (human immunodeficiency virus) reverse transcriptase enzyme. The same period also saw the emergence of biological or large molecule drugs (mainly proteins) to treat metabolic disease (e.g. recombinant insulin for diabetes; human growth hormone for dwarfism; interferon-beta for multiple sclerosis) and rare, orphan diseases (recombinant human deoxyribonuclease for cystic fibrosis). This period also witnessed the fulfilment of humanised and fully human monoclonal antibodies' promise in the treatment of cancer and other inflammatory diseases such as rheumatoid arthritis.

\section{The challenge in chronic disease: Targeting multiple target and pathways}

The success in conquering human disease has led to longer lives but has come with a price - the emergence of chronic indications associated with aging and longer lifespans. Thus, although there has been significant success in developing novel drugs with the single target paradigm, drug development in chronic diseases has increasingly been challenging. It is becoming clear that chronic diseases involve multiple, dysfunctional pathways that may need simultaneous modulation $[1,2]$. Most of the targets being modulated by current drugs or drugs in development are downstream of the disease pathway and correlate more with symptoms rather than the cause of the disease. Targets such as TNF (tumour necrosis factor)-alpha in inflammation and hyperglycaemia in diabetes are examples of this. Current trends in combination therapy and precision medicine in oncology with different immunotherapies and chemotherapies represent strategies in simultaneously targeting multiple pathways to manage disease.

Recent discoveries on the role of the microbiome have also led to a better understanding of its interaction with the host and its contribution to chronic diseases. Although it is possible that there are a limited number of control points which would have more broad and effective effects to manage disease, these are currently unknown. A key element in most chronic diseases is inflammation. Notwithstanding, therapeutic targets in inflammation remain vague and elusive due to multiple pathways. Not only is it important to identify which targets to address but it are also imperative to identify where in the pathway to intervene. In rheumatoid arthritis, the cause of inflammation in different joints was attributed to various pathways [3]. Another observation is crosstalk between rheumatoid arthritis and coronary artery disease within the same inflammation pathway [4]. Thus, modulating a single target or pathway associated with inflammation is probably not sufficient to manage or treat a chronic illness. Furthermore, the more upstream in the pathway the target is the better. Most targets of current drugs

Correspondence to: Leodevico L. Ilag, PhD, Xerion Limited, 246 Esplanade, Brighton, VIC 3186, Australia, E-mail: vilag@xerion.com.au

Received: January 09, 2018; Accepted: January 22, 2018; Published: January 26 2018 
such as TNF-alpha and IL (interleukin)-6 are downstream in the inflammation pathway.

\section{A new class of drugs and treatment paradigm in chronic disease: botanical extracts}

Clinical drug development is a rigorous process to determine the safety and efficacy of a drug candidate. One of the challenging steps in the process is determining the toxicity of drug candidates. Each candidate's toxicity profile has to be determined before embarking on any combination therapy. This increases the cost of developing combination therapies from novel drug candidates. Therefore, if one were to modulate multiple targets or pathways, only drugs that have gone through rigorous pre-clinical and clinical testing should be considered. In other words, each novel drug candidate has to undergo its own thorough toxicology testing.

For centuries, botanical extracts have been a source of treatment as demonstrated in Ayurveda (traditional Indian medicine) and Traditional Chinese Medicine (TCM). These traditional drugs are often extracts, derived from an entire plant, and are composed of a multitude of bioactive components. The fact that Ayurveda and TCM exist and remain popular attests to some efficacy albeit largely anecdotal. However, these therapeutic modalities lost favour with the pharmaceutical industry in decades past due to batch-to-batch inconsistencies in manufacturing and the lack of well-controlled clinical trials. The regulatory hurdles isolated these traditional medicines which had little beyond empirical evidence.

More recently, these traditional drugs are being tested in the same well-designed and controlled clinical studies applied to single entity drugs [5]. The US Food and Drug Administration (FDA) established a clear regulatory pathway for botanical drugs [6]. To date, only 2 drugs have been approved via this botanical path [5]. Veregen ${ }^{\circ}$ (sinecatechins), an extract derived from green tea (Camelia sinensis Kuntze) for treating genital and perianal warts topically was the first botanical drug approved by the US FDA in 2006. In 2012, the FDA approved the second botanical drug and first oral botanical drug, Fulyzaq (crofelemer) now called Mytesi ${ }^{\oplus}$, an anti-diarrheal drug for HIV patients derived from the latex of the South American croton tree (Croton lechlerii Müll. Arg).

The US FDA's botanical path considers the extract to be drug, and not the isolated chemical entities. The rationale behind this regulatory approach is that the observed efficacy has been associated with the use of the entire plant or its extracts. Failure to replicate therapeutic results can be attributed to the inability to isolate a distinct chemical entity that can reproduce the desired effects. The observed efficacy is thus associated with the action of multiple components including any synergistic effects that are lost during the fractionation of the extract. Botanical drug discovery will therefore involve methods that allow the isolation and characterisation of synergistic bioactive components and will play a significant role in sustaining efficacy in the development of this class of drugs [7].

A promising class of bioactive compounds in extracts are polyphenols; the two aforementioned FDA-approved botanical extracts involve polyphenols. Polyphenols and flavanols have multiple modes of action. They have shown efficacy towards managing metabolic disease, which can be attributed to these simultaneous activities [8]. These molecules are also known to regulate the microbiome [9]. Interestingly, polyphenols that are by-products of a filtered molasses concentrate has been shown to modulate postprandial glucose and insulin response.
Furthermore, from a clinical nutrition standpoint, these polyphenols can lower the glycemic index of carbohydrate-rich foods [10,11].

Several species of algae and microalgae (cyanobacteria) are a rich source of bioactive components that have shown potential for treating diabetes and metabolic syndrome [12]. Spirulina extracts have also shown novel, anti-microbial activity as well as anti-inflammatory properties that can treat mild to severe fungal infections such as onychomycosis [13], viral infections [14] and some effects on the microbiome [15].

Given the complex nature of a botanical extract, it is conceivable that it can simultaneously modulate multiple pathways of the host as well as the microbiome. This is a plausible reason for its observed efficacy. As an extract, it is most likely composed of multiple bioactive components involved in multiple mechanisms of action. However, complexity can potentially be a curse since the extract may have components with underlying toxic effects. In any case, the appropriate fractionation and separation techniques combined with analytical testing should provide a good handle in ensuring consistency in batchto-batch preparations.

Unlike single compounds that need to be tested individually, an advantage of the botanical regulatory path is that the extract can be tested as a single entity for toxicity and safety. It is not necessary to purify each component but there is a requirement that some components are identified and that there is a validated analytical assay that can be used to measure and ensure product consistency. Another advantage especially if the botanical is from a food source or considered as GRAS (generally recognized as safe), is its safety profile which expedites the conduct of clinical studies and can significantly reduce drug development costs.

For a botanical drug, the botanical source should be amenable to large-scale production and the extraction process needs to be scalable for drug development. Thus, one of the challenges is to ensure consistency in product batches which can be influenced by consistency in the growth environment of the botanical source. For example, microalgae can be cultured in bioreactors [16]. Advances in agricultural and aquaculture mass production techniques in controlled environments will help ensure product consistency, e.g. indoor agriculture/vertical farming [17]. Once the botanical material is produced, advanced analytical techniques in mass spectrometry and separation technologies provide the quality control needed for the botanical source and its extracts.

\section{A way forward - commercialisation strategy}

The new paradigm of targeting multiple pathways in chronic disease with botanical extracts represents a novel approach in treating or managing chronic diseases.

One possible commercialisation strategy, which allows early market entry, is to initially develop a nutritional product from a botanical source that is known to be safe or labelled GRAS with limited claims on the disease of interest. In addition to achieving market acceptance and early revenues, this approach allows the product to develop a safety profile and for the company developing it to accumulate efficacy data albeit anecdotal from customer feedback. At the same time, standard drug development via an IND (investigational new drug) can commence based on the US FDA botanical regulatory path, which requires an established manufacturing process and the conduct of well-controlled clinical trials to demonstrate efficacy and safety. This parallel development and marketing process allows cross-fertilisation of product information towards the development of a more efficacious 
and safe botanical drug. This approach provides a risk-mitigating and cost-effective strategy for future botanical drug development, which should translate to efficacious therapeutic options that lower healthcare costs especially for chronic diseases for any demographic and not only the aging population.

\section{Acknowledgement}

The author would like to thank Dr Jocelyn Ng for critical reading of the manuscript.

\section{References}

1. Van der Schyf CJ (2011) The use of multi-target drugs in the treatment of neurodegenerative diseases. Expert Rev Clin Pharmacol 4: 293-298. [Crossref]

2. Moneva MH, Dagogo-Jack S (2002) Multiple drug targets in the management of type 2 diabetes. Curr Drug Targets 3: 203-221. [Crossref]

3. Ai R, Hammaker D, Boyle DL, Morgan R, Walsh AM, et al. (2016) Joint-specific DNA methylation and transcriptome signatures in rheumatoid arthritis identify distinct pathogenic processes. Nat ICommun 7: 11849. [Crossref]

4. Niu X, Lu C, Xiao C, Zhang Z, Jiang M, et al. (2014) The Shared Crosstalk of Multiple Pathways Involved in the Inflammation between Rheumatoid Arthritis and Coronary Artery Disease Based on a Digital Gene Expression Profile. PLoS One 9: e113659. [Crossref]

5. Ahn K (2017) The worldwide trend of using botanical drugs and strategies for developing global drugs. BMB Rep 50: 111-116. [Crossref]

6. Botanical drug development guidance for industry. [https://www.fda.gov/downloads/ drugs/guidancecomplianceregulatoryinformation/guidances/ucm458484.pdf]

7. Junio HA, Sy-Cordero AA, Ettefagh KA, Burns JT, Micko KT, et al. (2011) SynergyDirected Fractionation of Botanical Medicines: A Case Study with Goldensea (Hydrastis canadensis). J Nat Prod 74: 1621-1629. [Crossref]
8. Hanhineva K, Törrönen R, Bondia-Pons I, Pekkinen J, Kolehmainen M, et al. (2010) Impact of dietary polyphenols on carbohydrate metabolism. Int J Mol Sci 11: 13651402. [Crossref]

9. Ozdal T, Sela DA, Xiao J, Boyacioglu D, Chen F, et al. (2016) The Reciproca Interactions between Polyphenols and Gut Microbiota and Effects on Bioaccessibility. Nutrients 8: 78-114. [Crossref]

10. Wright AG, Ellis TP, Ilag LL (2014) Filtered Molasses Concentrate from Sugar Cane: Natural Functional Ingredient Effective in Lowering the Glycaemic Index and Insulin Response of High Carbohydrate Foods. Plant Foods Hum Nutr 69: 310-316. [Crossref]

11. Ellis TP, Wright AG, Clifton PM, Ilag LL (2016) Postprandial insulin and glucose levels are reduced in healthy subjects when a standardised breakfast meal is supplemented with a filtered sugarcane molasses concentrate. Eur J Nutr 55: 2365-2376. [Crossref]

12. Ku CS, Yang Y, Park Y, Lee J (2013) Health Benefits of Blue-Green Algae: Prevention of Cardiovascular Disease and Nonalcoholic Fatty Liver Disease. J Med Food 16: 103111. [Crossref]

13. Parekh M, Ramaiah G, Pashilkar P, Ramanujam R, Johnston P. et al. (2017) A pilot single centre, double blind, placebo controlled, randomized, parallel study of Calmagen ${ }^{\circledR}$ dermaceutical cream and lotion for the topical treatment of tinea and onychomycosis. BMC Complement Alt Med 17: 46. [Crossref]

14. Chen YH, Chang GK, Kuo SM, Huang SY, Hu IC, et al. (2016) Well-tolerated Spirulina extract inhibits influenza virus replication and reduces virus-induced mortality. Sci Rep 6: 24253. [Crossref]

15. Neyrinck AM, Taminiau B, Walgrave H, Daube G, Cani PD, et al. (2017) Spirulina Protects against Hepatic Inflammation in Aging: An Effect Related to the Modulation of the Gut Microbiota? Nutrients 9: 633. [Crossref]

16. Yan N, Fan C, Chen Y, Hu Z (2016) The Potential for Microalgae as Bioreactors to Produce Pharmaceuticals. Int J Mol Sci 17. [Crossref]

17. Benke K, Tomkins B (2017) Future food-production systems: vertical farming and controlled-environment agriculture. Sustainability: Science, Practice and Policy 13: 13-26.

Copyright: C2018 Ilag LL. This is an open-access article distributed under the terms of the Creative Commons Attribution License, which permits unrestricted use, distribution, and reproduction in any medium, provided the original author and source are credited. 